Revista Iberoamericana, Vol. LXVIII, Núm. 198, Enero-Marzo 2002, 187-193

\title{
DE AFLICCIONES, TRAVESÍAS Y DESEOS: CONVERSACIÓN CON NOÉ JITRIK
}

\author{
POR \\ Pablo A. J. Brescia \\ University of California, Santa Barbara
}

Noé Jitrik (Argentina, 1928) parece estar siempre dispuesto a charlar sobre literatura. Durante su visita a la Universidad de California en Santa Bárbara, leyó un texto sugerente y divertido que llamó "Una selva de relatos". El magnetismo de sus palabras se explica por la singular mezcla de filosofía, teoría y creación literaria que lo hacen un escritor polifacético. Luego de varios años de residencia en México, Noé vive ahora en Argentina y es director en el del Instituto de Literatura Hispanoamericana de la Universidad de Buenos Aires. Autor de monografías críticas sobre escritores hispanoamericanos (Cristóbal Colón, Esteban Echeverría, José Hernández, Domingo F. Sarmiento, José Martí, Macedonio Fernández, Horacio Quiroga), de libros de teoría y crítica centrales para el estudio de la literatura latinoamericana como Procedimiento y mensaje en la novela (1962), El ochenta y su mundo (1968), El fuego de la especie (1971), El No-Existente Caballero (1975), Temas de teoría (1987), La vibración del presente (1987), La selva luminosa. Ensayos críticos 1987-1991 (1993) e Historia e imaginación literaria (1995) y de libros de creación literaria como Feriados y Díscola Cruz del Sur, iguíame! (poesía); La fisura mayor y Fin del ritual (relatos) y El ojo de jade y Mares del sur (novelas). Noé es uno de los nombres más reconocidos y reconocibles de la crítica y de la ficción latinoamericana de los últimos cuarenta años. Café y medialunas de por medio, nos sentamos a conversar sobre literatura argentina, sobre literatura latinoamericana y sobre Noé Jitrik.

Pablo Brescia: - Tomemos como punto de partida el tema de tu conferencia, la literatura argentina hoy. ¿Qué coordenadas pueden postularse para su análisis? Uno ve que el cierre que supone el fin de siglo amenaza el discurso crítico y entonces aparece una serie de adjetivos que se aplica a la literatura en general y también a la argentina en particular: literatura "posmoderna”, "apocalíptica", "desencantada". La pregunta es: ¿cómo ver la literatura argentina hoy?

NoÉ JITRIK: —En el plan de la Historia crítica de la literatura argentina que estamos coordinando, el último volumen se va a titular "Una literatura en aflicción”. O sea, la palabra aflicción es indicativa de un estado de ánimo de la literatura sobre sí misma después del largo proceso de su constitución. Es como si la literatura estuviera mirándose en su poder, en sus capacidades y también en sus limitaciones. Hay que considerar entonces las vetas que la cruzan para llevarla a ese estado. Estas vetas son de distinto tipo: 
por ejemplo, la experiencia de la dictadura, no en lo temático sino en relación con lo que pasa con la escritura misma, es una de ellas. Esta vertiente no está acabada ni siquiera en ese orden temático, en el sentido de una elaboración más refinada que la puramente testimonial o la puramente denuncialista. La travesía de las teorías literarias es otra de las vetas que hace que la literatura se reflexione, se mire en un espejo como para considerarse. Otra veta más son los modelos que entablan, podríamos decir, una lucha: el modelo tradicional, encarnado por la exitosa literatura comercial a la norteamericana y el modelo europeo de hacer literatura, a la francesa. Se podrían contraponer entonces las figuras de Raymond Carver y Paul Valéry como representativas de uno y otro eje. Así que ya tenemos por lo menos tres aspectos para empezar a entender lo que pasa con la literatura argentina actual. A todo esto se añade otra cosa: una especie de fervor, que se manifiesta sobre todo en la poesía.

PB: —¿Fervor de escritores, fervor de lectores?

NJ: - Fervor de escritores y me parece que también de lectores. Yo creo que el lector es una identidad desconocida y hacer la sociología de la lectura diciendo "sí se lee” o "no se lee” es sacarse el problema de encima. Se debe estar leyendo, porque de lo contrario no seguirían publicando las editoriales, no estaría tanta gente jugando sus cartas a la literatura y no sería la literatura un factor de “inmunización” contra un proceso de degradación cultural mundial que viene por el lado de estructuras económicas y políticas que aplastan diferencias. Entonces, y retomando la pregunta, nociones como "posmodernidad” o algo por el estilo no son, para mi gusto, instrumentos analíticos interesantes y todas estas otras vetas lo son mucho más.

PB: - Hablabas de un notable fervor por la poesía. ¿Cómo se explica esa efervescencia?

NJ: —El fervor por la poesía no sólo se nota en la profusión sino en los cambios cualitativos en el discurso poético. Hay poetas tradicionales y también jóvenes; hay un interés muy fuerte por el discurso poético en la academia. Por ejemplo, con el apoyo del Instituto de Literatura Hispanoamericana se publica una revista dedicada exclusivamente a poesía y problemas de teoría poética. Esto también viene ligado a la experiencia de la lectura de determinados poetas que han sido recuperados como importantes: Lamborghini, Gelman, Padeletti, Carrera, Kamenszain, por ejemplo, y luego los "nuevos" que van apareciendo. Hay un renovado vigor, entonces. Por eso cabría aclarar que la aflicción a la que se refiere el volumen en cuestión es un instrumento analítico para diferenciar este momento de otros: de la euforia de la constitución o la consagración o el éxito. Estamos ahora en una etapa de reconcentramiento que podría definir la literatura como una entidad con un perfil propio y actual.

PB: - Es decir, desde esta perspectiva se atiende a lo nuevo y se recupera una historia anterior.

NJ: —Exactamente, se recupera esa historia desde una integración más orgánica. Por ejemplo, el fenómeno Borges indica un orgullo y una orfandad, sentimientos que no creo que sean necesarios ya para entender el orden literario actual.

PB: - -Justamente hacia allá vamos. En 1999 se celebraron los cien años del nacimiento de Borges y hubo muchos fuegos artificiales. ¿Qué lugar va a ocupar Borges en la historia de la literatura argentina? Hay una frase de Nicolás Rosa en "Las sombras 
de Borges” que me parece pertinente aquí: "Donde más se quiere recordar más olvidamos. Lo que proponemos es simple y sencillo, pero costoso. Empecemos a olvidar a Borges”. Esto es de un artículo de 1986, el año de la muerte del autor de Ficciones. ¿Qué se juega en esta propuesta y cómo se combina con lo que decías en "Sentimientos complejos sobre Borges"?

NJ: - La culminación de Borges fue sustituir a los padres muertos. Es decir, tenemos ahora un gran padre, reconocido en el mundo entero. El triunfo de la literatura argentina encarnado en una persona; ésta es una manera de pensar que alude al hombre representativo del siglo xIX como lo imaginaba Sarmiento. Yo recuerdo precisamente cuándo Nicolás expresó esta frase: en una reunión sobre Borges en Rosario donde fuimos invitados Eduardo Grüner, él y yo. Esta idea de "matar" a Borges no debe ser entendida como iconoclastia clásica sino como espacialización. La literatura argentina se concibe como un espacio en el que hay inflexiones. Si la vemos así, Borges aparece en numerosos momentos. Ahí está: el olvido del que habla Nicolás es el de esa presencia edípica, que lo cubre todo y debajo de la cual nada o nadie importa.

PB: -O borra todo lo demás.

NJ: - Así es. Nos liberamos de eso para considerar dónde está Borges: Borges está en el desarrollo de la poesía argentina, Borges está en el descubrimiento de un lenguaje, Borges está en una inflexión crítica precursora de una cantidad de inquietudes teóricas. Pero ya no es el Borges superhéroe que elimina los compromisos y los riesgos que tiene la escritura, sino que se integra a ese mapa. En el primer volumen de nuestra Historia se ve que, de los veinte artículos que lo componen, Borges aparece convocado en seis o siete. Es decir, aparece en muchos lados, pero es memoria y olvido al mismo tiempo.

PB: - Hablando de literatura argentina y de Borges, mencionaste el proyecto de Historia de la Literatura Argentina. Cuéntanos un poco de este asunto. ¿Cómo surgió? ¿Qué se propone? ¿Llenar un vacío, renovar una serie de propuestas?

NJ: - Se diría que, desde el punto de vista editorial, se propone llenar un vacío. El proyecto surgió de la casa editorial y la intersección es paradójica: yo estaba trabajando sobre el concepto de historia de la literatura y pensando que habría que dejar de lado este tipo de género. Esta confluencia fue azarosa. Y hay que anotar cuestiones generacionales que importan en el contexto de nuestra historia reciente. Como la dictadura eliminó prácticamente a una generación, hay un hueco ahí. La gente que debía haber suplantado a la gente como yo, desapareció. Y gente como yo, está presente... por lo que hizo, pero también por esta razón: no hay mucha gente para determinado imaginario. Entonces, me convocan y yo me replanteo las cosas. La editorial podría haber llenado ese vacío haciendo una mezcla entre las historias de Rojas y Arrieta y los criterios de Anderson Imbert. Para mí, ésta era una ocasión de pensar de manera diferente: plantear una historia crítica que intentara integrar la idea de historicidad con la de crítica.

PB: -Quizá también sea una buena oportunidad para evitar la arrogancia de las "historias objetivas".

NJ: - Y para darle una inflexión que implique un compromiso que llamamos crítico: el punto de vista no será la objetividad científica a la manera del positivismo del siglo XIX, sino otro. Es decir, la idea de historia tiene que volver a ser puesta sobre la mesa. ¿Es una disciplina, una categoría? Para mí, es un relato. Y trata de ser un relato total. A partir de 
allí, elige el campo en el que se establece. El campo que se elegía en la época de Plutarco o Heródoto era el de las acciones mitológicas y políticas concretas que estaban dando nacimiento a la idea de los imperios. Ése es el criterio de selección. El de Plutarco, por la vía de los grandes conductores y el de Heródoto por la vía del desplazamiento, digamos. Con el paso de los siglos, son los criterios los que se revisan y los historiadores que innovan, innovan en eso. En las historias sociales del siglo xx, para hacer el relato se elige la vida cotidiana, el documento perdido, lo indirecto.

PB: - Hay una mayor exploración de lo privado y se ponen entre paréntesis los grandes acontecimientos públicos.

NJ: —Exactamente. Al mismo tiempo, la historia global elige zonas para formular su relato; de pronto se figura que lo global son determinadas particularidades que excluyen muchas otras. Para incluirlas, se hacen historias parciales que deberían confluir en la historia global. La vinculación se da por medio de los criterios. Si el criterio básico es que la historia es un relato y que hay que hacer el relato de la historia, un parcialidad en ese espectro es la literatura. Entonces hagamos el relato de la literatura entendida como historia. Lo cual nos lleva a zonas básicas de definición. ¿Cuáles son los momentos capitales del relato? Son los momentos de cambio, liberados de la noción de valor que justificaban las historias de la literatura anteriores, las cuales establecían cadenas de continuidades por valores atribuidos sin cuestionar el proceso de valoración. Parecía muy lógico que del Quijote se pasara al Lazarillo, ¿no? Mi idea no es excluir estos textos sino ubicarlos en este relato como momentos de una transición. En ese sentido, y para la Argentina, Borges representa ese tipo de momentos. Lo que importa de Borges es que es transicional; da lugar a capítulos nuevos. Había que ver cuáles eran los momentos para la literatura. Ya nos liberamos de criterios como las presidencias o los períodos políticos.

PB: - O sea, partir de la literatura para hacer el cruce. Es lo que comentaba Borges en su polémica con Roger Caillois sobre el inicio del género policial. Borges abogaba por un inicio puramente literario del relato policial (con "The Murders in the Rue Morgue" de Edgar Allan Poe en 1841) y Caillois lo derivaba de un hecho social (la aparición de la policía secreta de Fouché y la aprehensión que provoca esta circunstancia en la sociedad). Borges estaba más interesado en la serie literaria.

NJ: —Así es. La cuestión es buscar los cambios en el relato global que se llama literatura argentina; esos momentos dan lugar a capítulos y esos capítulos son concebidos como relatos. El primer volumen de nuestra historia se va a llamar "La irrupción de la crítica”. Esto se debe a que, en un momento determinado, las exigencias críticas cubren todo el terreno y se manifiestan en todas las expresiones literarias.

PB: -Leí un comentario tuyo al respecto en el que hablabas de no seguir criterios cronológicos.

NJ:- - Nunca hubiera empezado por el primer volumen porque eso hubiera creado un equívoco: lo que quería echar por la puerta habría vuelto por la ventana. En cambio, el volumen uno quizás sea el quinto o sexto que salga. Allí va a haber un trabajo teórico sobre la idea de una historia de la literatura que me parece coherente con la totalidad del proyecto.

PB: -Para cerrar el "capítulo" hablando de historia y de actualidad: ¿cómo colocarías la literatura argentina en el marco de la literatura latinoamericana? 
NJ: -Bueno, si hablé de tres vetas para entrar en este momento de la literatura argentina, la dimensión latinoamericana es una de esas calas. Debería ser entendida como problemática más que como sentimiento de integración a la manera de Henríquez Ureña. Lo de él es una cuenta pendiente, pero la cosa pasa por otro lado: lo que yo llamo travesías. Éstas implican apariciones y reapariciones de maneras de ver el trabajo literario que están casi en todas partes simultáneamente. La otra lectura es la comunicación interna que siempre fue sumamente productiva y muy fluida en América Latina: el viaje de Darío, el viaje de Borges, el viaje de Huidobro. Esos viajes son expresiones de la travesía; esto hace que aparezcan manifestaciones en América Latina que tienen más en común entre ellas de lo que se supone. Y no es necesariamente por obediencia a modelos comunes para todos. Es cierto que el libro de Octavio Paz, El festín de Esopo, se hace cargo del estructuralismo, que se manifiesta en Argentina, en Venezuela y en otros lugares. Al mismo tiempo, si eso germina es porque hay una necesidad de explicarse fenómenos literarios a los que el instrumento estructuralista les da alguna respuesta en un momento determinado. Lo mismo pasa con los fenómenos de la vanguardia: se pueden ver como un fervor renovador que estaba en todas partes y que indica, no que las sociedades ya han cambiado, sino que las sociedades desearían cambiar y que la literatura les ofrece un modelo para eso.

PB: - Es decir, sí hay una posibilidad de hablar de literatura latinoamericana.

NJ: - Yo creo que sí, con esta noción de travesías más que con la de integración. Esa palabra es muy furtiva. ¿En qué consiste? ¿En que todo sea igual? ¿En que haya ediciones de las mismas cosas en todas partes? Yo no puedo pretender que haya un Rulfo en la Argentina. Respeto el Rulfo mexicano porque hay un fruto de una larga maceración verbal y poética. Ahora, ¿qué tiene que ver Rulfo con algunas manifestaciones argentinas — que no son Rulfo-? Eso es lo que tenemos que ver si funciona o no. En la Argentina hay una tendencia a creer que la literatura argentina es muy autónoma, que no tiene nada que ver con la latinoamericana, por ejemplo con la vertiente del realismo mágico que es tan apreciada en otros lugares. Eso me parece bastante trivial como un modo de aislamiento; la singularidad no se refuerza por ausencia de lo que a otros les interesa. La singularidad es un punto de partida y lo que hay que ver son las resonancias que se pueden encontrar con otro tipo de fenómenos que se dan en el ámbito de la lengua, más que del continente.

PB: - Me gustaría pasar a tu labor como crítico y como escritor. Se puede pensar que el crítico se da cuenta que tiene una obra cuando empieza a ser recogida ¿no? Estoy pensando en este volumen reciente, Suspender toda certeza: antología crítica 1959-1976 [Buenos Aires, Biblos, 1997], y en otros volúmenes que recogen tus trabajos y trazan una trayectoria crítica. ¿Te sientes “canonizado" en la crítica? ¿Estar "canonizado” es estar petrificado?

NJ: —No, no me siento ni canonizado ni petrificado. Me parece — dicho de una manera un poco narcisista - que apenas se me está empezando a leer. Esto es un motivo de sorpresa permanente. Hace cosa de diez o doce años estaba en San Juan de Puerto Rico y una persona me habló sobre mi libro de Horacio Quiroga, escrito treinta años antes. Hay gente que lo ha recuperado como un instrumento crítico válido. Entonces, si eso ocurre con uno de mis primeros libros, imagínate con los demás. Tal vez esté empezando un proceso de acercamiento en el cual algunas de las cosas que hice pueden ser útiles como herramientas de trabajo. 
PB: - Y este fenómeno puede llevar a una aceleración que permita reconocer tus textos más nuevos.

NJ: - Claro, porque ahí sí hay una centripetización a partir de una llamada sobre algo pasado. Por ejemplo, en 1967 escribí un prólogo a una antología de textos del ochenta (siglo XIX) y eso pasó a otro lugar y se editó como libro suelto. Ahora se ha vuelto a editar de una manera modesta. Pero lo que pasa es que, de pronto, este libro se está leyendo como libro de historia. Incluso ahora, algunos periodistas políticos que hacen análisis de actualidad lo mencionan como referente del pasado que sirve para iluminar ciertos aspectos. Son trabajos que se abren paso muy lentamente. Ninguno de ellos busca ser canónico porque hay una permanente insatisfacción que me hace encarar las cosas siempre por primera vez. Estoy siempre en la posición inaugural.

PB: -Estos redescubrimientos de tus textos por parte del público lector, ¿te hace redescubrirlos a ti también?

NJ: —En parte sí. De pronto tengo que dar un curso, releo un trabajo mío y me digo: “no está mal, sigo suscribiendo estas ideas”. En estos días, en la Universidad de California en Irvine, tuve que exponer un análisis de un cuento de Rulfo que empecé a concebir en 1974 en El Colegio de México. Vi que no tenía otro modo de entrar a ese texto que la mirada que construí en ese entonces. No se gastó y está abierta todavía; incita a la lectura.

PB:- - Tu caso es significativo. Se puede hablar de un "escritor-crítico"; ese tándem en Latinoamérica es bastante frecuente por varias razones. En Argentina, Borges es el paradigma y en la actualidad están Piglia, Saer y otros, gente que trabaja con la ficción y la crítica. ¿Cómo funciona esta combinación para ti ? ¿Te pones un disfraz para escribir ficción y otro para escribir crítica o teoría, por ejemplo?

NJ: -En mi caso funciona en el orden del deseo, hasta casi por inspiración romántica. Neruda tiene un verso muy lindo: "De pronto me despierto en la noche pensando en el extremo sur”. Yo me despierto en la noche o en la mañana pensando en un verso que puedo escribir. En otras ocasiones me encuentro discutiendo un problema teórico y me digo que no lo puedo dejar perder. Otras veces siento la necesidad de narrar algo que estoy cargando y que espera su oportunidad de descarga. Se busca el instante de la explosión, del comienzo, pero hay algo que lo precede y que está en el orden del deseo. No establezco demasiadas diferencias en un campo o en otro. De ahí que me gustaría que mis trabajos críticos sean leídos como relatos y me gustaría que mis relatos sean integrados a mi pensamiento. El orden de la lectura no favorece esto, porque la lectura está muy acotada; publico una novela y las reseñas empiezan mal: "El crítico literario escribe una novela...” El lector entonces dice: “No voy a leer esta lata, esto va a ser una cosa teórica o una paráfrasis de los críticos de moda. Esto no es un relato”. A priori lo dejan de lado. Esto funciona entre los colegas también. Por ejemplo, los novelistas tienen una gran desconfianza en las narraciones que yo escribo, porque son de un teórico; los críticos alegan que escribo novelas y entonces no se puede ser muy serio; los poetas piensan que, como escribo novelas y crítica, no vale la pena leer mis poemas, que seguramente serán aburridos. Entonces, se piensa que la poesía es una relación exclusiva con lo inefable, el relato es una relación exclusiva con lo fático, la teoría es una relación exclusiva con lo intelectual. Esta persona no entra en ninguna categoría porque quiere entrar en todas. Para mí, no funciona así. Mi deseo me lleva a capturar una u otra cosa. 
PB: $-Y$, sin embargo, estos "detractores" parecerían desconocer una tradición de "practicantes-teóricos" en la historia de la literatura. Es decir, grandes escritores (Poe, Valéry, Borges) que se manejaban en esos dos registros.

NJ: - Claro. En la obra de Borges lo básico, cuantitativamente, es lo no narrativo. Sin embargo, de lo que se habla constantemente es de las ficciones. Borges se pasó la vida haciendo una crítica en forma de relato. Y la poesía la encaraba, a partir sobre todo de su segunda etapa, de manera cada vez más filosófica. A Borges se lo celebra al por mayor y se ignora este fenómeno que tiene que ver con el orden de la palabra: cómo la palabra puede dirigirse a lugares que no están preestablecidos.

PB: - Hablabas del proceso de tu escritura. Parece que lo tuyo entonces surge del deseo. ¿Cómo se va formando la práctica de la escritura desde un determinado punto de partida? ¿Es una tarea que hay que realizar todos los días, como una especie de disciplina? ¿O se recurre a destellos? Cortázar hablaba de otro verso de Neruda: "Mis criaturas nacen de un largo rechazo". Es decir, hay una especie de exorcismo, algo que hay que sacar y desarrollar.

NJ: - Yo me veo muy tranquilo, sin ninguna obligación. Es el ejercicio de algo que uno quiere hacer. Entonces, sentarme a escribir en mi caso puede ilustrarse con lo que llamaría una situación proustiana. Hay una escena en la que fijó su atención Ricardou: En un momento del relato, el narrador anda en auto por unas colinas que suben y bajan. Va hacia un pueblo. Y, de pronto, los campanarios de la iglesia se ven y desaparecen, se ven y desaparecen, creando un ritmo parecido al ritmo musical. "Cuando llegué allí”, dice el narrador, “entonces saqué lápiz y papel y empecé a componer”. Y lo que compone es lo que acabamos de leer. A mí eso me conmueve muchísimo; ese momento tiene un fondo teórico con respecto a la escritura que es muy importante. Y esto puede llegar a explicar mi propia relación compositiva. Desde luego, se crea también una solidaridad ética con lo que compongo y, por tanto, empiezo a tener una responsabilidad y una disciplina. Pero a veces me canso, me aburro. Yo escribo siempre tres o cuatro cosas al mismo tiempo.

PB: —Eres múltiple.

NJ: —Sí. Por ejemplo, ahora estoy escribiendo unos relatos que se van a llamar “Trayectos”, un trabajo sobre la poesía latinoamericana contemporánea, un trabajo sobre Borges y también estoy empezando una novela. Voy de acá para allá. Lo inicial fue resuelto con el "empezar a componer" de Proust. La siguiente etapa me hace seguir adelante aceptando el tedio, los momentos vacíos, como inherentes al proceso de la escritura. 